\title{
Method of principal factors estimation of optimal number of factors: an information criteria approach
}

\author{
Nwosu Dozie. F. ${ }^{1}$, Onyeagu Sidney. I. $^{2}$, Osuji George A. ${ }^{2}$ \\ ${ }^{1}$ Department of Mathematics and Statistics Federal Polytechnic Nekede, Owerri Imo State \\ ${ }^{2}$ Department of Statistics Nnamdi Azikiwe University Awka, Anambra State
}

\section{Email address:}

fedocon2003@yahoo.com(Nwosu D. F.)

\section{To cite this article:}

Nwosu Dozie. F., Onyeagu Sidney. I., Osuji George A.. Method of Principal Factors Estimation of Optimal Number of Factors: An Information Criteria Approach. American Journal of Theoretical and Applied Statistics. Vol. 2, No. 6, 2013, pp. 166-175. doi: 10.11648/j.ajtas.20130206.13

\begin{abstract}
The issue of the number of factors to be retained in a factor analysis has been an undefined. Be that as it may, this paper tries to x-ray the number of factors $(\mathrm{k})$ to be retained in a factor analysis for different sample sizes using the method of Principal Factor estimation when the number of variables are ten (10). Stimulated data were used for sample sizes of 30, 50 and 70 and the Akaike Information Criterion (AIC), the Schwarz Information Criterion (SIC) and the Hannan Quinne Information Criterion (HQIC) values were obtained when the number of factors(k) are two, three, and five (2,3 and 5). It was discorvered that the AIC, SIC, and HQIC values are smallest when $\mathrm{k}=5$, and highest when $\mathrm{k}=2$ for the sample sizes of 30 and 70. But, for a sample of 50, the values of these information criteria is smallest for $\mathrm{k}=3$, highest for $\mathrm{k}=5$. Hence, conclusion is drawn that for the sample sizes of 30 and 70, the optimal number of factors to retain is 5 and 3 for the sample size of 70 . This implies that, the number of factors to retain is a function of the sample size of the data.
\end{abstract}

Keywords: Factor Analysis, Factor Rotation, Principal Factors Estimation Method, Hannan Quinne Information Criteria, Akaike, Schwarz

\section{Introduction}

Factor analysis originated from the pioneering work on the subject by Charles Spearman (1904). It was developed extensively by L.L. Thurstone $(1931,1947)$. It is the most familiar multivariate procedure used in behavioural sciences. Some factors such as arguments over psychological interpretations of several early studies and lack of high speed computing facilities retarded its initial development as a statistical procedure. The purpose of factor analysis is to describe if possible the covariance relationships among variables in terms of a few underlying but unobservable random quantities called factors. Factor analysis can be considered as an extension of principal component analysis. Both are attempts to approximate the covariance matrix $\Sigma$, but the approximation based on factor analysis is more elaborate.

Lawley and Maxwell (1963) explained the differences between factor analysis and principal component analysis. Factor analysis is covariance (or correlation) oriented. In principal component analysis, all components are needed to reproduce an intercorrelation (covariance) exactly. In factor analysis, a few factors will reproduce the intercorrelation (covariances) exactly and model is fitted to data while in principal component analysis, a data is fitted to a model.

Factor analysis attempts to simplify complex and diverse relationships that exist among a set of observed variables by uncovering common dimensions or factors that link together the seemingly unrelated variables and consequently provides insight into the underlying structure of the data. The goal of factor analysis is to reduce a large number of variables to a smaller number of factors, to concisely describe the relationship among observed variables or to test theory about underlying processes(Onyeagu;2003).

\subsection{The Mathematical Model for Factor Structure}

Suppose that the multivariate system consists of $p$ responses described by the observable random variables $\mathrm{X}_{1}, \ldots, \mathrm{Xp}$. The Xi have a non-singular multinormal distribution. Since only the covariance structure will be of interest, we can assume without loss of generality that the population means of $X_{i}$ are zero(Harman;1976).

Let,

$$
\mathrm{Y}_{1}-\mu_{1}=\lambda_{11} \mathrm{X}_{1}+\cdots+\lambda_{1 \mathrm{~m}} \mathrm{X}_{m}+e_{1}
$$




$$
\mathrm{Y}_{\mathrm{p}}-\mu_{\mathrm{p}}=\lambda_{\mathrm{p} 1} \mathrm{X}_{1}+\cdots+\lambda_{\mathrm{pm}} \mathrm{X}_{m}+e_{\mathrm{p}}
$$

where,

$\mathrm{X}_{\mathrm{j}}=\mathrm{j}$-th common-factor variates

$\lambda_{\mathrm{ij}}=$ parameter reflecting importance of the $\mathrm{j}$-th factor in composition of ith response

$e_{\mathrm{i}}=\mathrm{i}^{\text {th }}$ specific factor variates.

In the language of factor analysis, $\lambda_{\mathrm{ij}}$ is called the loading of the ith response on the jth common factor.

For matrix version of the model;

Let,

$$
\begin{aligned}
& X^{I}=\left(x_{1}, x_{2}, \ldots, x_{m}\right), \quad y^{I}=\left(y_{1}, y_{2}, \ldots, y_{p}\right) \\
& \varepsilon^{I}=\left(\varepsilon_{1}, \varepsilon_{2}, \ldots, \varepsilon_{p}\right), \quad \mu^{I}=\left(\mu_{1}, \mu_{2}, \ldots, \mu_{p}\right),
\end{aligned}
$$$$
\text { and } \Lambda=\left[\begin{array}{ccc}
\lambda_{11} & . . & \lambda_{1 \mathrm{~m}} \\
: & : & : \\
\lambda_{\mathrm{p} 1} & . . & \lambda_{\mathrm{pm}}
\end{array}\right] \text {. }
$$

Then, the factor model can be written as

$$
\left(y_{\mathrm{i}}-\mu_{\mathrm{y}}\right)=\Lambda \mathrm{X}_{\mathrm{i}}+\varepsilon_{\mathrm{i}}, \mathrm{i}=1, \ldots, \mathrm{q} .
$$

where $q$ is the number of observations. $Y_{i}$ is an observed vector with $\mathrm{p}$ components with mean $\mu_{\mathrm{y}}$ and $\Lambda$ is a $p \times q$ matrix called the factor loadings. $\mathrm{X}_{\mathrm{i}}$ is an observed vector with $q$ components, $q<p$, the components of which are called common factors and $\varepsilon_{\mathrm{i}}$ is an unobserved error with mean $0_{(\mathrm{px} 1)}$ and is called the specific factor. $\mathrm{X}_{\mathrm{i}}$ is assumed to follow a normal distribution with

$\mathrm{E}\left(\mathrm{X}_{\mathrm{i}}\right)=0_{(\mathrm{q} \times 1)}, \quad \operatorname{Var}\left(\mathrm{X}_{\mathrm{i}}\right)=\mathrm{E}\left(\mathrm{X}_{\mathrm{i}} \mathrm{X}_{\mathrm{i}}^{\mathrm{I}}\right)=\mathrm{I}_{(\mathrm{q} \times \mathrm{q})}$, that is the orthogonal case.

Then, $\mathrm{Y}_{\mathrm{i}} / \mathrm{X}_{\mathrm{i}}$ follows $N\left(\mu_{\mathrm{y}}+\Lambda \mathrm{X}_{\mathrm{i}}, \operatorname{Cov}\left(\varepsilon_{\mathrm{i}}\right)\right)$, where $\operatorname{Cov}\left(\varepsilon_{\mathrm{i}}\right)=\psi^{2}=\operatorname{diag}\left(\psi_{1}^{2}, \ldots, \psi_{\mathrm{p}}^{2}\right)$.

$\psi^{2}$ is called uniqueness or specific variance(Bai and Serena; 2002).

Consequently, a covariance structure for $\mathrm{Y}$ is

$$
\begin{aligned}
& \begin{aligned}
\Sigma= & \operatorname{Cov}\left(\mathrm{Y}_{\mathrm{i}}\right)=\mathrm{E}\left(Y_{\mathrm{i}}-\mu_{\mathrm{y}}\right)\left(Y_{\mathrm{i}}-\mu_{\mathrm{y}}\right)^{I} \\
= & \mathrm{E}\left(\Lambda \mathrm{X}_{\mathrm{i}}+\varepsilon_{\mathrm{i}}\right)\left(\Lambda \mathrm{X}_{\mathrm{i}}+\varepsilon_{\mathrm{i}}\right)^{I} \\
& =\mathrm{E}\left(\Lambda \mathrm{X}_{\mathrm{i}} \mathrm{X}_{\mathrm{i}}^{\mathrm{I}} \Lambda^{I}+\varepsilon_{\mathrm{i}} \mathrm{X}_{\mathrm{i}}^{\mathrm{I}} \Lambda^{I}+\Lambda \mathrm{X}_{\mathrm{i}} \varepsilon_{\mathrm{i}}^{\mathrm{I}}+\varepsilon_{\mathrm{i}} \varepsilon_{\mathrm{i}}^{\mathrm{I}}\right) \\
& =\mathrm{E}\left(\Lambda \mathrm{X}_{\mathrm{i}} \mathrm{X}_{\mathrm{i}}^{\mathrm{I}} \Lambda^{I}\right)+\mathrm{E}\left(\varepsilon_{\mathrm{i}} \mathrm{X}_{\mathrm{i}}^{\mathrm{I}} \Lambda^{I}\right)+\mathrm{E}\left(\Lambda \mathrm{X}_{\mathrm{i}} \varepsilon_{\mathrm{i}}^{\mathrm{I}}\right)+\mathrm{E}\left(\varepsilon_{\mathrm{i}} \varepsilon_{\mathrm{i}}^{\mathrm{I}}\right)
\end{aligned}
\end{aligned}
$$

Recall that, $\mathrm{E}\left(\mathrm{X}_{\mathrm{i}}\right)=0_{(\mathrm{q} \times 1)}, \quad \mathrm{E}\left(\mathrm{X}_{\mathrm{i}} \mathrm{X}_{\mathrm{i}}^{\mathrm{I}}\right)=\mathrm{I}_{(\mathrm{qxq})}$, $\operatorname{Cov}\left(\varepsilon_{\mathrm{i}}\right)=\mathrm{E}\left(\varepsilon_{\mathrm{i}} \varepsilon_{\mathrm{i}}^{\mathrm{I}}\right)=\psi^{2}$,

Then,

$$
\mathrm{E}\left(\Lambda \mathrm{X}_{\mathrm{i}} \mathrm{X}_{\mathrm{i}}^{\mathrm{I}} \Lambda^{I}\right)+\mathrm{E}\left(\varepsilon_{\mathrm{i}} \mathrm{X}_{\mathrm{i}}^{\mathrm{I}} \Lambda^{I}\right)+\mathrm{E}\left(\Lambda \mathrm{X}_{\mathrm{i}} \varepsilon_{\mathrm{i}}^{I}\right)+\mathrm{E}\left(\varepsilon_{\mathrm{i}} \varepsilon_{\mathrm{i}}^{I}\right)
$$

$=\Lambda \Lambda^{I}+\psi^{2}$, and

$\operatorname{Cov}\left(Y_{\mathrm{i}}, \mathrm{X}_{\mathrm{i}}\right)=\mathrm{E}\left(Y_{\mathrm{i}}-\mu_{\mathrm{y}}\right) \mathrm{X}_{\mathrm{i}}^{\mathrm{I}}=\mathrm{E}\left(\Lambda \mathrm{X}_{\mathrm{i}}+\varepsilon_{\mathrm{i}}\right) \mathrm{X}_{\mathrm{i}}^{\mathrm{I}}$.

Introducing communality, $\operatorname{Cov}\left(\mathrm{Y}_{\mathrm{i}}\right)=\Lambda \Lambda^{I}+\psi^{2}$ can be written as

$$
\begin{gathered}
\operatorname{Var}\left(Y_{i j}\right)=\Lambda_{1 j}^{2}+\ldots+\Lambda_{q j}^{2}+\Psi_{j}^{2} ; \operatorname{Cov}\left(Y_{i j}, Y_{i k}\right)= \\
\Lambda_{1 j} \Lambda_{1 k}+\ldots+\Lambda_{q j} \Lambda_{q k} \text { and } \operatorname{Cov}\left(Y_{i k}, X_{j k}\right)=\Lambda_{j k} .
\end{gathered}
$$

Communality is the portion of the variance of the variable contributed by the $\mathrm{q}$ common factors.

Suppose the $\mathrm{jth}$ communality is $\mathrm{h}_{j}^{2}$, then

$\operatorname{Var}\left(Y_{\mathrm{ij}}\right)=\sigma_{\mathrm{ij}}=$ communality + specific variance $=$ $\mathrm{h}_{\mathrm{j}}^{2}+\psi_{\mathrm{j}}=\left(\lambda_{1 \mathrm{j}}^{2}+\lambda_{2 \mathrm{j}}^{2}+\cdots+\lambda_{\mathrm{qj}}^{2}\right)+\psi_{\mathrm{j}}, \mathrm{j}=1, \ldots, \mathrm{p}$.

The $\mathrm{j}$-th communality is the sum of square of the loading of the $j$ th variable on the $q$ common factor. When the number of factors $\mathrm{q}>1$, there are multiple factor loadings that generate the same covariance matrix.

The loading in the model above can be multiplied by an orthogonal matrix without impairing their ability to reproduce the covariance matrix in $\Sigma=\Lambda \Lambda^{\mathrm{I}}+\psi^{2}$.

Let $\beta$ be any qxq orthogonal matrix. If we let $\Lambda^{*}=\Lambda \beta$ and $\mathrm{X}_{\mathrm{i}}^{*}=\beta^{\mathrm{I}} \mathrm{X}_{\mathrm{i}}$, then $\mathrm{X}_{\mathrm{i}}^{*}$ has the same statistical properties as $X_{i}$ since

$$
\begin{gathered}
\mathrm{E}\left(\mathrm{X}_{\mathrm{i}}^{*}\right)=E\left(\beta^{\mathrm{I}} \mathrm{X}_{\mathrm{i}}\right)=\beta^{\mathrm{I}} E\left(\mathrm{X}_{\mathrm{i}}\right)=0 . \\
\operatorname{Cov}\left(\mathrm{X}_{\mathrm{i}}^{*}\right)=\operatorname{Cov}\left(\beta^{\mathrm{I}} \mathrm{X}_{\mathrm{i}}\right)=\beta^{\mathrm{I}} \operatorname{Cov}\left(\mathrm{X}_{\mathrm{i}}\right) \beta=\beta^{\mathrm{I}} \beta=\mathrm{I}_{\mathrm{mxm}}
\end{gathered}
$$

$\Lambda$ and $\Lambda^{\mathrm{I}}$ yield the same covariance because $\Lambda \Lambda^{\mathrm{I}}=\Lambda^{*} \Lambda^{* I}$. The factor model

$$
\left(Y_{\mathrm{i}}-\mu_{\mathrm{y}}\right)=\Lambda \mathrm{X}_{\mathrm{i}}+\varepsilon_{\mathrm{i}}=\Lambda \beta \beta^{\mathrm{I}} \mathrm{X}_{\mathrm{i}}+\varepsilon_{\mathrm{i}}=\Lambda^{*} \mathrm{X}_{\mathrm{i}}^{*}+\varepsilon_{\mathrm{i}},
$$

produces the same covariance matrix $\Sigma$, since $\Sigma=\Lambda \Lambda^{\mathrm{I}}+$ $\psi^{2}=\Lambda \beta \beta^{\mathrm{I}} \Lambda+\psi^{2}=\Lambda^{*} \Lambda^{* \mathrm{I}}+\psi^{2}$.

A particular set of loadings needs to be chosen. A good set is one that is easily interpreted. This means sparse solution with many zero. Factor rotation is one approval to finding the solution, as it rotates the coordinates system for $\mathrm{Y}$ on X(Johnson and Wichern;2002) .

In this work, we consider four kinds of orthogonal factor rotations namely, varimas, equimax, quartimax and orthomax.

\section{The Principal Factors Method}

The principal factors method (also called the principal axis method) uses an initial estimate $\widehat{\Psi}$ and factors $S-$ $\widehat{\Psi}$ or $\mathrm{R}-\widehat{\Psi}$ to obtain

$$
\begin{aligned}
& S-\widehat{\Psi} \cong \widehat{\Lambda} \widehat{\Lambda}^{I}, \\
& R-\widehat{\Psi} \cong \widehat{\Lambda} \widehat{\Lambda}^{I} .
\end{aligned}
$$

where $\widehat{\Lambda}$ is pxm and is calculated using the eigenvalues and eigenvectors of $S-\widehat{\Psi}$ or $R-\widehat{\Psi}$.

The i-th diagonal element of $S-\widehat{\Psi}$ is given by $S_{i i}-\widehat{\Psi}_{i}$, which is the $\mathrm{i}$-th communality $\quad \hat{h}_{\mathrm{i}}^{2}=$ $S_{i i}-\widehat{\Psi}_{i}$. Likewise, the diagonal elements of $R-\widehat{\Psi}$ are the communalities $\hat{h}_{\mathrm{i}}^{2}=1-\widehat{\Psi}_{i}$. (Clearly, $\widehat{\Psi}_{i}$ and $\hat{h}_{\mathrm{i}}^{2}$ have different values for $\mathrm{S}$ than for $\mathrm{R}$ ). With these diagonal values, $S-\widehat{\Psi}$ and $R-\widehat{\Psi}$ have the form 


$$
\begin{aligned}
S-\widehat{\Psi} & =\left[\begin{array}{ccll}
\hat{h}_{1}^{2} & S_{12} & \ldots & S_{1 p} \\
S_{21} & \hat{h}_{2}^{2} & \ldots & S_{2 p} \\
\vdots & \vdots & & \vdots \\
\vdots & \vdots & & \vdots \\
S_{p 1} & S_{p 2} & \ldots & \hat{h}_{\mathrm{p}}^{2}
\end{array}\right] \\
R-\widehat{\Psi} & =\left[\begin{array}{cccc}
\hat{h}_{1}^{2} & r_{12} & \ldots & r_{1 p} \\
r_{21} & \hat{h}_{2}^{2} & \ldots & r_{2 p} \\
\vdots & \vdots & & \vdots \\
\vdots & \vdots & & \vdots \\
r_{p 1} & r_{p 2} & \ldots & \hat{h}_{\mathrm{p}}^{2}
\end{array}\right]
\end{aligned}
$$

A popular initial estimate for a communality in $R-\widehat{\Psi}$ is $\hat{h}_{\mathrm{i}}^{2}=R_{\mathrm{i}}^{2}$, the squared multiple correlation between $y_{i}$ and the other $p-1$ variables. This can be found as

$$
\hat{h}_{\mathrm{i}}^{2}=R_{\mathrm{i}}^{2}=1-\frac{1}{r^{i i}},
$$

where, $r^{i i}$ is the i-th diagonal element of $R^{-1}$.

For $S-\widehat{\Psi}$, an initial estimate of the communality is

$$
\hat{h}_{\mathrm{i}}^{2}=S_{i i}-\frac{1}{S^{i i}}
$$

where $S_{i i}$ is the i-th diagonal element of $S$ and $s^{i i}$ is the i-th diagonal element of $S^{-1}$.

It can be shown that equation (2.6) is equivalent to

$$
\hat{h}_{\mathrm{i}}^{2}=S_{i i}-\frac{1}{S^{i i}}=S_{i i} R_{\mathrm{i}}^{2}
$$

which is a reasonable estimate of the amount of variance that $y_{i}$ has in common with the other y's to use equation (2.5) or (2.6), $R$ or $S$ must be non-singular. If $R$ is singular, we can use the absolute value or the square of the largest correlation in the $\mathrm{i}$-th row of $R$ as an estimate of communality.

After obtaining communality estimates, we calculate eigenvalues and eigenvectors of $S-\widehat{\Psi}$ or $R-\widehat{\Psi}$ and use it to obtain estimates of factor loadings, $\widehat{\Lambda}$. Then, the columns and rows of $\widehat{\Lambda}$ can be used to obtain new eigenvalues (variance explained) and communalities, respectively. The sum of squares of the j-th column of $\widehat{\Lambda}$ is the j-th eigenvalues of $S-\widehat{\Psi}$ or $R-\widehat{\Psi}$, and the sum of squares of the $\mathrm{i}$-th row of $\widehat{\Lambda}$ is the communality of $y_{i}$. The proportion of variance explained by the $j$-th factor is

$$
\frac{\sum_{i=1}^{p} \widehat{\lambda}_{\mathrm{ij}}^{2}}{\operatorname{tr}(S-\widehat{\Psi})}=\frac{\theta_{j}}{\sum_{i=1}^{p} \theta_{i}}, \text { or } \frac{\sum_{i=1}^{p} \widehat{\lambda}_{\mathrm{ij}}^{2}}{\operatorname{tr}(R-\widehat{\Psi})}=\frac{\theta_{j}}{\sum_{i=1}^{p} \theta_{i}},
$$

where $\theta_{j}$ is the $\mathrm{j}^{\text {th }}$ eigenvalue of $S-\widehat{\Psi}$ or $R-\widehat{\Psi}$. The matrices $S-\widehat{\Psi}$ and $R-\widehat{\Psi}$ are not necessarily positive semi-definite and will often have some small negative eigenvalues. In such a case, the cumulative proportion of variance will exceed 1 and then decline to 1 as the negative eigenvalues are added.

\subsection{Information Criteria}

The necessity of introducing the concept of model evaluation has been recognized as one of the important technical areas and the problem is posed on the choice of the best approximating model among a class of competing models by a suitable model evaluation criterion given a data set. Model evaluation criteria are figures of merit, or performance measures for competing models. Factor analysis can be characterized as multivariate technique for analyzing the internal relationship among a set of variables. Based on the usual factor analysis model, choosing a model with too few parameters can involve making unrealistically simple assumptions and lead to high bias, poor prediction, and missed opportunities for insight. Such models are not flexible enough to describe the sample or the population well. A model with too many parameters can fit the observed data very well, but be too closely tailored to it; such models may generalize poorly. Penalized-likelihood information criteria, such as Akaike's information criterion (AIC), the Schwarz's information criterion (SIC), the Hannan-Quinn information criterion (HQIC) and so on are widely used for model selection. The comparison of the models using information criterion can be viewed as equivalent to a likelihood ratio test and understanding the differences among the criteria may make it easier to compare the results and to use them to make informed decisions(Akaike;1973).

AKAIKE'S information criterion is probably the most relevant and famous as for the comparison and selection between different models and is constructed on log likelihood

$$
\mathrm{AIC}=-2 \log \max L+2 k
$$

where L denotes the likelihood function of the factor model and $\mathrm{k}$ is the number of the model's parameter/factors. $\log \max L(k)=-\frac{1}{2} N|\log | \Sigma_{k}\left|+\operatorname{tr} \Sigma_{k}^{-1} S\right|$, where $\mathrm{S}$ denotes the sample covariance matrix of $\mathrm{Y}$ and $\Sigma_{k}=$ $\Lambda_{k} \Lambda_{k}^{-1}+\psi^{2} ; \Lambda_{k}$ is the matrix factor of factor loading. The first term can be interpreted as a goodness-of-fit measurement, while the second gives a growing penalty to increasing numbers of parameters according to the parsimony principle. In the choice of model, a minimization rule is used to select the model with the minimum Akaike information criterion value.

Still in the likelihood based procedures, Schwarz (1978) proposed the alternative information criterion given by

$$
S I C=-\log \max L+\frac{1}{2} k \log N .
$$

Unlike the AIC, SIC considers the number of $\mathrm{N}$ of observations and is therefore less favourable to factors inclusion.

Finally, the third criteria is the Hannan-Quinn information criterion (HQC); it is a criterion for model selection. It is an alternative to Akaike information criterion (AIC) and Bayesian information criterion (BIC). It is given as $\quad H Q I C=-\log \max L+2 k \log \log N$

where $\mathrm{k}$ is the number of parameters, $\mathrm{N}$ is the number of observations. 


\section{Comparison of AIC and SIC after Rotation at Different Sample Sizes and Different Retained Number of Factors (k)}

3.1. For $n=30, p=10$ and $k=2$

3.1.1. Rotated Factor Loadings

\begin{tabular}{llllllll}
\multicolumn{2}{l}{ Varimax Loadings } & \multicolumn{2}{l}{ Equamax Loadings } & \multicolumn{2}{c}{ Quartimax Loadings } & \multicolumn{2}{c}{ Orthomax Loadings } \\
I & II & I & II & I & II & I & II \\
\hline-0.0642 & -0.0629 & -0.0674 & -0.0595 & -0.635 & -0.0636 & -0.0830 & 0.0344 \\
0.0653 & 0.5438 & 0.0938 & 0.5396 & 0.0595 & 0.5445 & 0.2639 & -0.4800 \\
-0.2413 & 0.2374 & -0.2285 & 0.2498 & -0.2438 & 0.2348 & -0.1350 & -0.3104 \\
0.4135 & 0.0049 & 0.4131 & -0.0169 & 0.4134 & 0.0093 & 0.3853 & 0.1501 \\
0.3448 & -0.4505 & 0.3206 & -0.4680 & 0.3496 & -0.4468 & 0.1513 & 0.5468 \\
-0.7154 & -0.0663 & -0.7179 & -0.0285 & -0.7147 & -0.0739 & -0.6883 & -0.2060 \\
0.2980 & 0.3957 & 0.3185 & 0.3795 & 0.2938 & 0.3989 & 0.4244 & -0.2555 \\
-0.0381 & -0.3340 & -0.0556 & -0.3316 & -0.0345 & -0.3344 & -0.1603 & 0.2956 \\
0.0809 & 0.1253 & 0.0874 & 0.1208 & 0.0795 & 0.1261 & 0.1219 & -0.0859 \\
0.5046 & -0.1780 & 0.4945 & -0.2043 & 0.5064 & -0.1726 & 0.4014 & 0.3537 \\
\hline
\end{tabular}

\subsubsection{Factor Rotation Matrix}

\begin{tabular}{|c|c|c|}
\hline & Factor I & Factor II \\
\hline $\begin{array}{l}\text { Factor I } \\
\text { Factor II }\end{array}$ & $\begin{array}{l}0.9798 \\
0.2002\end{array}$ & $\begin{array}{r}-0.2002 \\
0.9798\end{array}$ \\
\hline \multicolumn{3}{|c|}{ Equamax. } \\
\hline & Factor I & Factor II \\
\hline $\begin{array}{l}\text { Factor I } \\
\text { Factor II }\end{array}$ & $\begin{array}{l}0.9679 \\
0.2515\end{array}$ & $\begin{array}{r}-0.2515 \\
0.9679\end{array}$ \\
\hline \multicolumn{3}{|c|}{ Quartimax. } \\
\hline & Factor I & Factor II \\
\hline $\begin{array}{l}\text { Factor I } \\
\text { Factor II }\end{array}$ & $\begin{array}{lr}0.9818 \\
0.1897\end{array}$ & $\begin{array}{l}0.1897 \\
0.9818\end{array}$ \\
\hline
\end{tabular}

\begin{tabular}{|c|c|c|}
\hline \multicolumn{3}{|r|}{ Factor II } \\
\hline $\begin{array}{l}\text { Factor I } \\
\text { Factor II }\end{array}$ & $\begin{array}{ll}0.8338 & 0 \\
0.5520 & \\
\end{array}$ & $\begin{array}{l}5520 \\
0.9679\end{array}$ \\
\hline \multicolumn{3}{|c|}{ 3.1.3. Information Criteria } \\
\hline Information Criteria & & Values \\
\hline Log Likelihood & & -118.6680 \\
\hline Akaike & & 241.3360 \\
\hline Schwarz & & 120.1451 \\
\hline Hannan Quinne & & 119.3457 \\
\hline
\end{tabular}

\subsection{For $n=30, p=10$ and $k=3$}

\subsubsection{Rotated Factor Loadings}

\begin{tabular}{|c|c|c|c|c|c|c|c|c|c|c|c|}
\hline \multicolumn{3}{|c|}{ Varimax Loadings } & \multicolumn{3}{|c|}{ Equamax Loadings } & \multicolumn{3}{|c|}{ Quartimax Loadings } & \multicolumn{3}{|c|}{ Orthomax Loadings } \\
\hline $\begin{array}{l}\text { Factor } \\
\text { I }\end{array}$ & $\begin{array}{l}\text { Factor } \\
\text { II }\end{array}$ & $\begin{array}{l}\text { Factor } \\
\text { III }\end{array}$ & $\begin{array}{l}\text { Factor } \\
\text { I }\end{array}$ & $\begin{array}{l}\text { Factor } \\
\text { II }\end{array}$ & $\begin{array}{l}\text { Factor } \\
\text { III }\end{array}$ & $\begin{array}{l}\text { Factor } \\
\text { I }\end{array}$ & $\begin{array}{l}\text { Factor } \\
\text { II }\end{array}$ & $\begin{array}{l}\text { Factor } \\
\text { III }\end{array}$ & $\begin{array}{l}\text { Factor } \\
\text { I }\end{array}$ & $\begin{array}{l}\text { Factor } \\
\text { II }\end{array}$ & $\begin{array}{l}\text { Factor } \\
\text { III }\end{array}$ \\
\hline 0.0957 & -0.1784 & 0.2864 & 0.1452 & -0.2688 & -0.1723 & 0.0910 & -0.1776 & 0.2884 & 0.1452 & -0.2688 & -0.1723 \\
\hline-0.0268 & 0.5787 & 0.0116 & -0.0437 & -0.0126 & 0.5776 & -0.0256 & 0.5788 & 0.0092 & -0.0437 & -0.0126 & 0.5776 \\
\hline-0.0066 & 0.0331 & 0.5721 & 0.0820 & -0.5659 & 0.0388 & -0.0150 & 0.0351 & 0.5718 & 0.0820 & -0.5659 & 0.0388 \\
\hline 0.2283 & 0.1722 & -0.4317 & 0.1521 & 0.4631 & 0.1749 & 0.2351 & 0.1702 & -0.4289 & 0.1521 & 0.4631 & 0.1749 \\
\hline 0.2336 & -0.3113 & -0.4441 & 0.1713 & 0.4736 & -0.3083 & 0.2395 & -0.3133 & -0.4395 & 0.1713 & 0.436 & -0.3083 \\
\hline-0.7385 & -0.1067 & 0.0927 & -0.7110 & -0.2079 & -0.1291 & -0.7401 & -0.1046 & 0.0820 & -0.7110 & -0.2079 & -0.1291 \\
\hline 0.2666 & 0.4172 & 0.0182 & 0.2522 & 0.0261 & 0.4256 & 0.2672 & 0.4166 & 0.0207 & 0.2522 & 0.0261 & 0.4256 \\
\hline-0.0190 & -0.3286 & -0.0766 & -0.0199 & 0.0709 & -0.3298 & -0.0186 & -0.3288 & -0.0757 & -0.0199 & 0.0709 & -0.3298 \\
\hline 0.0704 & 0.1313 & 0.0078 & 0.0664 & 0.0040 & 0.1335 & 0.0706 & 0.1312 & 0.0084 & 0.0664 & 0.0041 & 0.1335 \\
\hline 0.6056 & -0.1949 & 0.0099 & 0.6058 & 0.0841 & -0.1755 & 0.64049 & -0.1963 & 0.0196 & 0.6058 & 0.0841 & -0.1755 \\
\hline
\end{tabular}




\subsubsection{Factor Rotation Matrix}

Varimax.

\begin{tabular}{llcc}
\hline & Factor I & Factor II & Factor III \\
\hline Factor I & 0.8742 & -0.0274 & -0.4848 \\
Factor II & 0.1791 & 0.9462 & 0.2694 \\
Factor III & 0.4513 & -0.3224 & 0.8321 \\
\hline
\end{tabular}

Equamax.

\begin{tabular}{llll}
\hline & Factor I & Factor II & Factor III \\
\hline Factor I & 0.8483 & -0.0142 & -0.5293 \\
Factor II & 0.1827 & 0.9461 & 0.2674 \\
Factor III & 0.4970 & -0.3235 & 0.8052 \\
\hline
\end{tabular}

\subsection{For $n=30, p=10$, and $k=5$}

Quartimax.

\begin{tabular}{llcc}
\hline & Factor I & Factor II & Factor III \\
Factor I & 0.8813 & -0.0311 & -0.4715 \\
Factor II & 0.1772 & 0.9467 & 0.2689 \\
Factor III & 0.4380 & -0.3205 & 0.8399 \\
\hline
\end{tabular}

Orthomax

\begin{tabular}{lccc}
\hline & Factor I & Factor II & Factor III \\
\hline Factor I & 0.7880 & 0.6156 & -0.0047 \\
Factor II & 0.1877 & -0.2330 & 0.9542 \\
Factor III & 0.5863 & -0.7528 & -0.2992 \\
\hline
\end{tabular}

\subsubsection{Information Criteria}

\begin{tabular}{ll}
\hline Information Criteria & Values \\
\hline Log Likelihood & -115.2820 \\
Akaike & 236.5640 \\
Schwarz & 117.4977 \\
Hannan Quinne & 116.2985 \\
\hline
\end{tabular}

\subsubsection{Rotated Factor Loadings}

\begin{tabular}{|c|c|c|c|c|c|c|c|c|c|}
\hline \multicolumn{5}{|c|}{ Varimax Loadings } & \multicolumn{5}{|c|}{ Equamax Loadings } \\
\hline 1 & 2 & 3 & 4 & 5 & 1 & 2 & 3 & 4 & 5 \\
\hline 0.0291 & -0.2467 & 0.3084 & 0.0620 & 0.2325 & 0.0477 & 0.1511 & -0.1059 & -0.1428 & 0.3975 \\
\hline 0.0234 & -0.0180 & -0.6165 & 0.0890 & 0.0394 & 0.0330 & 0.1354 & -0.2761 & 0.0460 & -0.5410 \\
\hline-0.0010 & -0.5785 & -0.0341 & 0.0792 & -0.0162 & 0.0512 & 0.0799 & -0.2646 & -0.5112 & 0.0488 \\
\hline 0.2259 & 0.4472 & -0.1390 & -0.0010 & 0.1133 & 0.1857 & 0.0571 & 0.1099 & 0.4582 & -0.1530 \\
\hline 0.1903 & 0.4352 & 0.3914 & 0.1877 & -0.2053 & 0.1227 & 0.0440 & 0.6111 & 0.2002 & 0.1600 \\
\hline-0.7466 & -0.0988 & 0.0735 & -0.0342 & -0.0851 & -0.7327 & -0.1018 & -0.0455 & -0.1659 & 0.0636 \\
\hline 0.2088 & 0.0397 & -0.2198 & 0.2673 & 0.3851 & 0.1995 & 0.4346 & -0.2040 & 0.1871 & -0.0892 \\
\hline 0.0375 & 0.0550 & 0.3189 & -0.5019 & -0.0406 & 0.0588 & -0.4707 & -0.0673 & 0.1305 & 0.1752 \\
\hline-0.0117 & 0.0244 & 0.0899 & 0.4600 & 0.0840 & -0.0421 & 0.4389 & 0.1693 & -0.0548 & 0.0382 \\
\hline 0.6235 & -0.0267 & 0.1618 & -0.0660 & -0.0882 & 0.6206 & -0.0825 & 0.1496 & -0.0291 & 0.1121 \\
\hline \multicolumn{5}{|c|}{ Quartimax Loadings } & \multicolumn{5}{|c|}{ Orthomax Loadings } \\
\hline 1 & 2 & 3 & 4 & 5 & 1 & 2 & 3 & 4 & 5 \\
\hline 0.0297 & 0.2638 & -0.2609 & 0.0756 & 0.2653 & 0.0240 & -0.2256 & 0.3701 & 0.0538 & 0.1527 \\
\hline 0.0282 & -0.6139 & 0.0174 & 0.1016 & -0.0465 & 0.0099 & -0.0751 & -0.5901 & 0.0568 & 0.1823 \\
\hline-0.0109 & -0.0656 & -0.5769 & 0.0696 & -0.0181 & 0.0186 & -0.5746 & 0.0084 & 0.1026 & -0.0351 \\
\hline 0.2374 & -0.1219 & 0.4528 & 0.0167 & 0.0814 & 0.1988 & 0.4317 & -0.1460 & -0.0393 & 0.1858 \\
\hline 0.1906 & 0.4459 & 0.3998 & 0.1655 & -0.1837 & 0.1925 & 0.4915 & 0.2963 & 0.2133 & -0.2102 \\
\hline-0.7508 & 0.0701 & -0.0906 & -0.0391 & -0.0507 & -0.7323 & -0.1100 & 0.0621 & -0.0190 & -0.1685 \\
\hline 0.2225 & -0.2530 & 0.0526 & 0.3057 & 0.3216 & 0.1698 & 0.0120 & -0.1353 & 0.2029 & 0.4741 \\
\hline 0.0342 & 0.1329 & 0.0539 & -0.5051 & 0.0226 & 0.0493 & 0.0564 & 0.1293 & -0.4871 & -0.1330 \\
\hline-0.0073 & 0.0925 & 0.0131 & 0.4649 & 0.0507 & -0.0253 & 0.0414 & 0.0968 & 0.4467 & 0.1274 \\
\hline 0.6190 & 0.1724 & -0.0483 & -0.0819 & -0.0763 & 0.6324 & 0.0103 & 0.1397 & -0.0423 & -0.0803 \\
\hline
\end{tabular}

\subsubsection{Factor Rotation Matrix}

Varimax.

\begin{tabular}{llllll}
\hline & Factor 1 & Factor 2 & Factor 3 & Factor 4 & Factor 5 \\
\hline Factor 1 & 0.8580 & 0.4939 & 0.1058 & 0.0858 & 0.0374 \\
Factor 2 & 0.1716 & -0.2264 & -0.7907 & 0.4076 & 0.3579 \\
Factor 3 & 0.4338 & -0.8274 & 0.3469 & -0.0354 & 0.0751 \\
Factor 4 & -0.1857 & 0.0665 & 0.4480 & 0.8605 & 0.1412 \\
Factor 5 & -0.1086 & 0.1255 & 0.2064 & -0.2914 & 0.9192 \\
\hline
\end{tabular}

Equamax

\begin{tabular}{llcccc}
\hline & Factor 1 & Factor 2 & Factor 3 & Factor 4 & Factor 5 \\
\hline Factor 1 & 0.7969 & 0.1087 & 0.3833 & 0.4540 & 0.0018 \\
Factor 2 & 0.1911 & 0.5859 & -0.5280 & -0.0276 & -0.5837 \\
Factor 3 & 0.5083 & 0.0221 & -0.2768 & -0.6656 & 0.4706 \\
Factor 4 & -0.2496 & 0.7894 & 0.4476 & -0.1300 & 0.3119 \\
Factor 5 & -0.0877 & 0.1459 & -0.5453 & 0.5771 & 0.5836 \\
\hline
\end{tabular}

Quartimax.

\begin{tabular}{llcccc}
\hline & Factor 1 & Factor 2 & Factor 3 & Factor 4 & Factor 5 \\
\hline Factor 1 & 0.8669 & 0.1400 & 0.4702 & 0.0877 & 0.0117 \\
Factor 2 & 0.1839 & -0.8282 & -0.1816 & 0.4480 & 0.2159 \\
Factor 3 & 0.4191 & 0.2856 & -0.8516 & -0.0485 & 0.1231 \\
Factor 4 & -0.1791 & 0.4521 & 0.0313 & 0.8653 & 0.1178 \\
Factor 5 & -0.0836 & 0.0923 & 0.1402 & -0.2015 & 0.9614 \\
\hline
\end{tabular}

Orthomax.

\begin{tabular}{lccccc}
\hline & Factor 1 & Factor 2 & Factor 3 & Factor 4 & Factor 5 \\
\hline Factor 1 & 0.6376 & 0.4172 & 0.6419 & 0.0820 & 0.0246 \\
Factor 2 & 0.1114 & -0.4246 & 0.2291 & -0.6661 & 0.5578 \\
Factor 3 & 0.5581 & -0.5949 & -0.2381 & 0.5081 & 0.1402 \\
Factor 4 & -0.2275 & 0.3484 & -0.0820 & 0.3943 & 0.8153 \\
Factor 5 & -0.4666 & -0.4127 & 0.6871 & 0.3686 & -0.0630 \\
\hline
\end{tabular}


3.3.3. Information Criteria

\begin{tabular}{ll}
\hline Information Criteria & Values \\
\hline Log Likelihood & -111.6615 \\
Akaike & 233.3230 \\
Schwarz & 115.3543 \\
Hannan Quinne & 113.3557 \\
\hline
\end{tabular}

\subsection{For $n=50, p=10$, and $k=2$}

3.4.1. Rotated Factor Loadings

\begin{tabular}{|c|c|c|c|c|c|c|c|}
\hline \multicolumn{2}{|c|}{ Varimax Loadings } & \multicolumn{2}{|c|}{ Equamax Loadings } & \multicolumn{2}{|c|}{ Quartimax Loadings } & \multicolumn{2}{|c|}{ Orthomax Loadings } \\
\hline I & II & I & II & I & II & I & II \\
\hline 0.4485 & 0.4337 & 0.3588 & 0.5103 & 0.4600 & 0.4213 & -0.1449 & 0.6068 \\
\hline-0.6198 & 0.0535 & -0.6188 & -0.0641 & -0.6182 & 0.0703 & 0.5513 & -0.2883 \\
\hline-0.0483 & 0.2891 & -0.1019 & 0.2748 & -0.0405 & 0.2903 & 0.1962 & 0.2177 \\
\hline-0.0766 & -0.0221 & -0.0711 & -0.0361 & -0.0772 & -0.0200 & 0.0527 & -0.0598 \\
\hline-0.1126 & -0.1679 & -0.0790 & -0.1861 & -0.1171 & -0.1648 & 0.0046 & -0.2021 \\
\hline 0.2855 & -0.0914 & 0.2976 & -0.0360 & 0.2830 & -0.0991 & -0.2899 & 0.0765 \\
\hline 0.5097 & 0.0521 & 0.4908 & 0.1471 & 0.5110 & 0.0383 & -0.4017 & 0.3181 \\
\hline 0.1060 & -0.4813 & 0.1947 & -0.4527 & 0.0929 & -0.4840 & -0.3482 & -0.3488 \\
\hline-0.2930 & 0.2081 & -0.3270 & 0.1492 & -0.2873 & 0.2159 & 0.3590 & 0.0178 \\
\hline 0.0519 & 0.3253 & -0.0102 & 0.3292 & 0.0607 & 0.3237 & 0.1311 & 0.3021 \\
\hline
\end{tabular}

\subsubsection{Factor Rotation Matrix}

Varimax.

\begin{tabular}{lll}
\hline & Factor I & Factor II \\
\hline Factor I & 0.9806 & 0.1960 \\
Factor II & -0.1960 & 0.9806 \\
\hline
\end{tabular}

Equamax

\begin{tabular}{lll}
\hline & Factor I & Factor II \\
\hline Factor I & 0.9262 & 0.3771 \\
Factor II & -0.3771 & 0.9262 \\
\hline
\end{tabular}

Quartimax.

\begin{tabular}{|c|c|c|}
\hline & Factor I & Factor II \\
\hline Factor I & 0.9855 & 0.1694 \\
\hline Factor II & -0.1694 & 0.9855 \\
\hline \multicolumn{3}{|c|}{ Orthomax } \\
\hline & Factor I & Factor II \\
\hline Factor I & -0.7213 & 0.6926 \\
\hline Factor II & $\begin{array}{ll}0.6926 & 0\end{array}$ & 213 \\
\hline
\end{tabular}

\subsubsection{Information Criteria}

\begin{tabular}{ll}
\hline Information Criteria & Values \\
\hline Log Likelihood & -239.8450 \\
Akaike & 483.6900 \\
Schwarz & 241.5440 \\
Hannan Quinne & 240.7657 \\
\hline
\end{tabular}

\subsection{For $n=50, p=10$, and $k=3$}

\subsubsection{Rotated Factor Loadings}

\begin{tabular}{|c|c|c|c|c|c|c|c|c|c|c|c|}
\hline \multicolumn{3}{|c|}{ Varimax Loadings } & \multicolumn{3}{|c|}{ Equamax Loadings } & \multicolumn{3}{|c|}{ Quartimax Loadings } & \multicolumn{3}{|c|}{ Orthomax Loadings } \\
\hline $\begin{array}{l}\text { Factor } \\
\text { I }\end{array}$ & $\begin{array}{l}\text { Factor } \\
\text { II }\end{array}$ & $\begin{array}{l}\text { Factor } \\
\text { III }\end{array}$ & $\begin{array}{l}\text { Factor } \\
\text { I }\end{array}$ & $\begin{array}{l}\text { Factor } \\
\text { II }\end{array}$ & $\begin{array}{l}\text { Factor } \\
\text { III }\end{array}$ & $\begin{array}{l}\text { Factor } \\
\text { I }\end{array}$ & $\begin{array}{l}\text { Factor } \\
\text { II }\end{array}$ & $\begin{array}{l}\text { Factor } \\
\text { III }\end{array}$ & $\begin{array}{l}\text { Factor } \\
\text { I }\end{array}$ & $\begin{array}{l}\text { Factor } \\
\text { II }\end{array}$ & $\begin{array}{l}\text { Factor } \\
\text { III }\end{array}$ \\
\hline 0.6151 & 0.2322 & 0.0738 & 0.6213 & -0.0074 & 0.2273 & 0.6091 & 0.2376 & 0.1010 & 0.5339 & -0.0567 & 0.3865 \\
\hline-0.6020 & 0.2385 & 0.1382 & -0.5759 & 0.2211 & 0.2406 & -0.6097 & 0.2349 & 0.1073 & -0.5917 & 0.2883 & 0.0714 \\
\hline-0.0674 & 0.2570 & 0.1277 & 0.0861 & 0.1204 & 0.2539 & 0.0593 & 0.2585 & 0.1285 & 0.0277 & 0.1242 & 0.2659 \\
\hline 0.0799 & -0.1549 & 0.3391 & 0.1237 & 0.3234 & -0.1549 & 0.0647 & -0.1514 & 0.3439 & 0.1959 & 0.3012 & -0.1275 \\
\hline-0.0866 & -0.1962 & 0.1414 & -0.0683 & 0.1495 & -0.1974 & -0.0918 & -0.1957 & 0.1388 & 0.0032 & 0.1453 & -0.2118 \\
\hline 0.1547 & -0.0731 & -0.2839 & 0.1144 & -0.3031 & -0.0705 & 0.1687 & -0.0742 & -0.2756 & 0.0956 & -0.3160 & -0.0302 \\
\hline 0.3851 & 0.0279 & -03733 & 0.3313 & -0.4216 & 0.0302 & 0.4022 & 0.0278 & -0.3548 & 0.2539 & -0.4500 & 0.1278 \\
\hline 0.0137 & -0.5326 & -0.0158 & 0.0072 & -0.0236 & -0.5325 & 0.0184 & -0.5326 & -0.0104 & 0.1458 & -0.0511 & -0.5101 \\
\hline-0.0598 & 0.1192 & 0.4453 & 0.0019 & 0.4506 & 0.1140 & -0.0819 & 0.1225 & 0.4409 & 0.0201 & 0.4535 & 0.1002 \\
\hline 0.1197 & 0.3169 & -0.0032 & 0.1207 & -0.0157 & 0.3161 & 0.1173 & 0.3177 & -0.0003 & 0.0299 & -0.0115 & 0.3372 \\
\hline
\end{tabular}




\subsubsection{Factor Rotation Matrix}

\begin{tabular}{llll}
\multicolumn{3}{c}{ Varimax. } \\
\hline & Factor I & Factor II & Factor III \\
\hline Factor I & 0.8989 & 0.0180 & -0.4378 \\
Factor II & 0.1582 & 0.9184 & 0.3626 \\
Factor III & 0.4087 & -0.3952 & 0.8227 \\
\hline
\end{tabular}

\subsection{For $n=30, p=10$, and $k=5$}

Quartimax.

\begin{tabular}{|c|c|c|c|}
\hline & Factor I & Factor II & Factor III \\
\hline Factor I & 0.9186 & 0.0214 & -0.3946 \\
\hline Factor II & 0.1339 & 0.9227 & 0.3616 \\
\hline Factor III & 0.3718 & -0.3850 & 0.8447 \\
\hline \multicolumn{4}{|c|}{ Orthomax } \\
\hline & Factor I & Factor II & Factor III \\
\hline Factor I & 0.7320 & -0.6323 & 0.2538 \\
\hline Factor II & -0.0009 & 0.3716 & 0.9284 \\
\hline Factor III & 0.6813 & 0.6798 & -0.2714 \\
\hline
\end{tabular}

\subsubsection{Information Criteria}

\begin{tabular}{ll}
\hline Information Criteria & Values \\
\hline Log Likelihood & -236.4975 \\
Akaike & 478.9950 \\
Schwarz & 239.0460 \\
Hannan Quinne & 237.8786 \\
\hline
\end{tabular}

\subsubsection{Rotated Factor Loadings}

\begin{tabular}{|c|c|c|c|c|c|c|c|c|c|}
\hline \multicolumn{5}{|c|}{ Varimax Loadings } & \multicolumn{5}{|c|}{ Equamax Loadings } \\
\hline 1 & 2 & 3 & 4 & 5 & 1 & 2 & 3 & 4 & 5 \\
\hline 0.5901 & 0.2836 & 0.0242 & 0.1131 & 0.0711 & 0.5819 & 0.2639 & -0.0608 & 0.0645 & 0.1762 \\
\hline-0.6315 & 0.2194 & 0.0819 & 0.0653 & 0.0445 & -0.6066 & 0.2276 & 0.1530 & 0.1275 & -0.0223 \\
\hline 0.0172 & 0.1732 & 0.0989 & 0.1316 & 0.3041 & 0.0007 & 0.1458 & 0.0919 & 0.1382 & 0.3180 \\
\hline 0.0288 & -0.0887 & 0.0930 & 0.4435 & 0.0077 & 0.0685 & -0.0911 & 0.0309 & 0.4473 & 0.0114 \\
\hline-0.0492 & -0.0470 & 0.0735 & 0.0489 & -0.4135 & 0.0194 & -0.0106 & 0.0536 & 0.0647 & -0.4194 \\
\hline 0.1370 & 0.0345 & -0.4148 & 0.0481 & -0.0581 & 0.0877 & 0.0364 & -0.4335 & -0.0129 & -0.0245 \\
\hline 0.3890 & -0.0033 & -0.2925 & -0.1813 & 0.1472 & 0.3089 & -0.0233 & -0.3060 & -0.2483 & 0.2033 \\
\hline 0.0364 & -0.5667 & -0.0207 & 0.0549 & -0.0385 & 0.0362 & -0.5621 & -0.0343 & 0.0486 & -0.0809 \\
\hline-0.0668 & 0.1290 & 0.4106 & 0.1767 & -0.0671 & 0.0133 & 0.1350 & 0.3862 & 0.2300 & -0.0700 \\
\hline 0.1335 & 0.2413 & 0.1637 & -0.2005 & 0.1088 & 0.1281 & 0.2289 & 0.1751 & -0.1908 & 0.1378 \\
\hline \multicolumn{5}{|c|}{ Quartimax Loadings } & \multicolumn{5}{|c|}{ Orthomax Loadings } \\
\hline 1 & 2 & 3 & 4 & 5 & 1 & 2 & 3 & 4 & 5 \\
\hline 0.5883 & 0.2846 & 0.0478 & 0.1236 & 0.0489 & 0.2976 & 0.5461 & -0.1804 & 0.0347 & -0.1629 \\
\hline-0.6340 & 0.2212 & 0.0561 & 0.0558 & 0.0527 & -0.5633 & -0.2496 & -0.1453 & 0.1811 & 0.1625 \\
\hline 0.0183 & 0.1818 & 0.1008 & 0.1321 & 0.2982 & -0.1145 & 0.1416 & -0.0763 & 0.2698 & -0.1953 \\
\hline 0.0182 & -0.0907 & 0.1028 & 0.4415 & 0.0085 & -0.0910 & 0.2516 & 0.3001 & 0.1996 & 0.1125 \\
\hline-0.0625 & -0.0585 & 0.0725 & 0.0452 & -0.4108 & 0.0481 & -0.0025 & 0.0394 & -0.1220 & 0.4058 \\
\hline 0.1513 & 0.0292 & -0.4083 & 0.0581 & -0.0624 & -0.0590 & 0.1861 & 0.0674 & -0.3681 & -0.1399 \\
\hline 0.4065 & -0.0004 & -0.2798 & -0.1694 & 0.1385 & 0.2466 & 0.1424 & -0.0472 & -0.2612 & -0.3740 \\
\hline 0.0353 & -0.5681 & -0.0140 & 0.0518 & -0.0231 & 0.2099 & -0.1933 & 0.4938 & -0.0440 & 0.0159 \\
\hline-0.0874 & 0.1291 & 0.4097 & 0.1685 & -0.0695 & -0.0181 & 0.0835 & -0.0813 & 0.3664 & 0.2779 \\
\hline 0.1326 & 0.2468 & 0.1634 & -0.1995 & 0.0992 & 0.1319 & 0.0308 & -0.3346 & 0.1247 & -0.0949 \\
\hline
\end{tabular}

\subsubsection{Factor Rotation Matrix}

Varimax.

\begin{tabular}{llllll}
\hline & Factor 1 & Factor 2 & Factor 3 & Factor 4 & Factor 5 \\
\hline Factor 1 & 0.9023 & 0.0291 & -0.3708 & -0.1568 & 0.1513 \\
Factor 2 & 0.1000 & 0.8444 & 0.4161 & 0.0547 & 0.3175 \\
Factor 3 & 0.3858 & -0.2759 & 0.5520 & 0.6485 & -0.2229 \\
Factor 4 & -0.1486 & -0.1712 & -0.2775 & 0.5186 & 0.7764 \\
Factor 5 & -0.0699 & 0.4250 & -0.5547 & 0.5319 & -0.4731 \\
\hline
\end{tabular}

Equamax.

\begin{tabular}{lllccc}
\hline & Factor 1 & Factor 2 & Factor 3 & Factor 4 & Factor 5 \\
\hline Factor 1 & 0.8020 & -0.0020 & -0.4499 & -0.2768 & 0.2787 \\
Factor 2 & 0.1250 & 0.8117 & 0.4044 & 0.0933 & 0.3915 \\
Factor 3 & 0.5241 & -0.2659 & 0.4007 & 0.6763 & -0.1913 \\
Factor 4 & -0.2540 & -0.2355 & -0.2865 & 0.4860 & 0.7495 \\
Factor 5 & -0.0439 & 0.4637 & -0.6256 & 0.4701 & -0.4132 \\
\hline
\end{tabular}

Quartimax.

\begin{tabular}{llcccc}
\hline & Factor 1 & Factor 2 & Factor 3 & Factor 4 & Factor 5 \\
\hline Factor 1 & 0.9220 & 0.0311 & -0.3372 & -0.1355 & 0.12975 \\
Factor 2 & 0.0899 & 0.8559 & 0.4144 & 0.0550 & 0.2906 \\
Factor 3 & 0.3479 & -0.2825 & 0.5808 & 0.6412 & -0.2253 \\
Factor 4 & -0.1275 & -0.1544 & -0.2719 & 0.5226 & 0.7829 \\
Factor 5 & -0.0671 & 0.4034 & -0.5507 & 0.5426 & -0.4848 \\
\hline
\end{tabular}


Orthomax

\begin{tabular}{llcccc}
\hline & Factor 1 & Factor 2 & Factor 3 & Factor 4 & Factor 5 \\
\hline Factor 1 & 0.5874 & 0.4895 & -0.0547 & -0.3691 & -0.5255 \\
Factor 2 & -0.1591 & 0.4020 & -0.7224 & 0.5301 & -0.1005 \\
Factor 3 & 0.3569 & 0.4681 & 0.4711 & 0.4792 & 0.4494 \\
Factor 4 & -0.4676 & 0.1567 & 0.5023 & 0.3035 & -0.6421 \\
Factor 5 & -0.5326 & 0.5959 & 0.0302 & -0.5108 & 0.3154 \\
\hline
\end{tabular}

3.6.3. Information Criteria

\begin{tabular}{ll}
\hline Information Criteria & Values \\
\hline Log Likelihood & -237.0900 \\
Akaike & 484.1800 \\
Schwarz & 241.3374 \\
Hannan Quinne & 239.3919 \\
\hline
\end{tabular}

3.7. For $n=70, p=10$ and $k=2$

3.7.1. Rotated Factor Loadings

\begin{tabular}{llllllll}
\hline \multicolumn{2}{l}{ Varimax Loadings } & \multicolumn{2}{l}{ Equamax Loadings } & \multicolumn{2}{l}{ Quartimax Loadings } & \multicolumn{2}{l}{ Orthomax Loadings } \\
$\mathbf{I}$ & II & I & II & I & II & I & II \\
\hline 0.4699 & 0.1452 & 0.4705 & 0.1433 & 0.4698 & 0.1456 & 0.4434 & -0.2128 \\
-0.2739 & 0.1140 & -0.2735 & 0.1151 & -0.2740 & 0.1138 & -0.1235 & 0.2698 \\
0.1437 & -0.0249 & 0.1436 & -0.0254 & 0.1437 & -0.0248 & 0.0885 & -0.1159 \\
-0.0327 & -0.3481 & -0.0341 & -0.3480 & -0.0324 & -0.3482 & -0.2606 & -0.2332 \\
-0.4369 & 0.0296 & -0.4368 & 0.0313 & -0.4369 & 0.0292 & -0.3005 & 0.3186 \\
-0.1192 & 0.0560 & -0.1189 & 0.0564 & -0.1192 & 0.0559 & -0.0494 & 0.1220 \\
-0.0112 & 0.3385 & -0.0098 & 0.3385 & -0.0114 & 0.3385 & 0.2218 & 0.2559 \\
0.2034 & -0.1007 & 0.2030 & -0.1015 & 0.2035 & -0.1005 & 0.0808 & -0.2121 \\
0.0872 & 0.3954 & 0.0888 & 0.3950 & 0.0869 & 0.3955 & 0.3327 & 0.2308 \\
0.2853 & -0.1387 & 0.2847 & -0.1399 & 0.2854 & -0.1385 & 0.1150 & -0.2956 \\
\hline
\end{tabular}

\subsubsection{Factor Rotation Matrix}

Varimax.

\begin{tabular}{lll}
\hline & Factor I & Factor II \\
\hline Factor I & 0.9998 & -0.0213 \\
Factor II & 0.0213 & 0.9998 \\
\hline
\end{tabular}

Equamax.

\begin{tabular}{lll}
\hline & Factor I & Factor II \\
\hline Factor I & 0.9997 & -0.0253 \\
Factor II & 0.0253 & 0.9997 \\
\hline
\end{tabular}

3.8. For $n=70, p=10$, and $k=3$
Quartimax.

\begin{tabular}{|c|c|}
\hline & Factor II \\
\hline Factor I & -0.0205 \\
\hline Factor II & 0.0205 \\
\hline \multicolumn{2}{|r|}{ Orthomax } \\
\hline & Factor I Factor II \\
\hline Factor I & $0.7191 \quad-0.6950$ \\
\hline Factor II & $0.6950 \quad 0.7191$ \\
\hline
\end{tabular}

\subsubsection{Information Criteria}

\begin{tabular}{ll}
\hline Information Criteria & Values \\
\hline Log Likelihood & -328.3934 \\
Akaike & 660.7868 \\
Schwarz & 330.2385 \\
Hannan Quinne & 329.4575 \\
\hline
\end{tabular}

\subsubsection{Rotated Factor Loadings}

\begin{tabular}{|c|c|c|c|c|c|c|c|c|c|c|c|}
\hline \multicolumn{3}{|c|}{ Varimax Loadings } & \multicolumn{3}{|c|}{ Equamax Loadings } & \multicolumn{3}{|c|}{ Quartimax Loadings } & \multicolumn{3}{|c|}{ Orthomax Loadings } \\
\hline $\begin{array}{l}\text { Factor } \\
\text { I }\end{array}$ & $\begin{array}{l}\text { Factor } \\
\text { II }\end{array}$ & $\begin{array}{l}\text { Factor } \\
\text { III }\end{array}$ & $\begin{array}{l}\text { Factor } \\
\text { I } \\
\end{array}$ & $\begin{array}{l}\text { Factor } \\
\text { II }\end{array}$ & $\begin{array}{l}\text { Factor } \\
\text { III }\end{array}$ & $\begin{array}{l}\text { Factor } \\
\text { I } \\
\end{array}$ & $\begin{array}{l}\text { Factor } \\
\text { II }\end{array}$ & $\begin{array}{l}\text { Factor } \\
\text { III }\end{array}$ & $\begin{array}{l}\text { Factor } \\
\text { I } \\
\end{array}$ & $\begin{array}{l}\text { Factor } \\
\text { II }\end{array}$ & $\begin{array}{l}\text { Factor } \\
\text { III }\end{array}$ \\
\hline 0.5157 & 0.0368 & -0.0268 & 0.5142 & 0.0452 & 0.0401 & 0.5145 & 0.0353 & -0.0464 & 0.4769 & 0.1988 & 0.0326 \\
\hline-0.2715 & 0.1597 & -0.0664 & -0.2615 & -0.0967 & 0.1609 & -0.2735 & 0.1596 & -0.0579 & -0.2436 & -0.1108 & 0.1789 \\
\hline 0.0644 & 0.0974 & 0.2220 & 0.0327 & 0.2322 & 0.0890 & 0.0729 & 0.0995 & 0.2184 & -0.0390 & 0.2477 & 0.0070 \\
\hline-0.0462 & -0.3815 & 0.0461 & -0.0483 & 0.0249 & -0.3832 & -0.0451 & -0.3809 & 0.0517 & -0.0100 & -0.1118 & -0.3704 \\
\hline-0.4095 & 0.0333 & -0.1545 & -0.3849 & -0.2075 & 0.0375 & -0.4149 & 0.0327 & -0.1395 & -0.3194 & -0.2850 & 0.0972 \\
\hline-0.0771 & 0.0033 & -0.1287 & -0.0589 & -0.1378 & 0.0079 & -0.0819 & 0.0021 & -0.1258 & -0.0215 & -0.1390 & 0.0523 \\
\hline-0.0272 & 0.4278 & 0.0172 & -0.0334 & 0.0295 & 0.4267 & -0.0257 & 0.4281 & 0.0138 & -0.0874 & 0.1518 & 0.3917 \\
\hline 0.1354 & -0.0185 & 0.2108 & 0.1057 & 0.2264 & -0.0261 & 0.1432 & -0.0167 & 0.2058 & 0.0453 & 0.2268 & -0.0981 \\
\hline 0.2221 & 0.2129 & -0.3325 & 0.2632 & -0.2908 & 0.2267 & 0.2099 & 0.2090 & -0.3427 & 0.3027 & -0.1177 & 0.3158 \\
\hline 0.1512 & 0.0466 & 0.3894 & 0.0964 & 0.4078 & 0.0320 & 0.1658 & 0.0502 & 0.3830 & -0.0173 & 0.4068 & -0.1041 \\
\hline
\end{tabular}




\subsubsection{Factor Rotation Matrix}

\begin{tabular}{llll}
\multicolumn{3}{c}{ Varimax. } \\
\hline & Factor I & Factor II & Factor III \\
\hline Factor I & 0.9246 & 0.0005 & 0.3809 \\
Factor II & 0.2148 & 0.8250 & -0.5227 \\
Factor III & -0.3145 & 0.5651 & 0.7627 \\
\hline
\end{tabular}

\subsection{For $n=70, p=10$, and $k=5$.}

Quartimax.

\begin{tabular}{|c|c|c|c|}
\hline & Factor I & Factor II & Factor III \\
\hline Factor I & 0.9382 & 0.0022 & 0.3460 \\
\hline Factor II & 0.1968 & 0.8192 & -0.5387 \\
\hline Factor III & -0.2846 & 0.5735 & 0.7681 \\
\hline \multicolumn{4}{|c|}{ Orthomax. } \\
\hline & Factor I & Factor II & Factor III \\
\hline Factor I & 0.6990 & 0.6968 & -0.1609 \\
\hline Factor II & 0.2890 & -0.0695 & 0.9548 \\
\hline Factor III & -0.6541 & 0.7139 & 0.2499 \\
\hline
\end{tabular}

3.8.3. Information Criteria.

\begin{tabular}{ll}
\hline Information Criteria & Values \\
\hline Log Likelihood & -326.2455 \\
Akaike & 656.4910 \\
Schwarz & 329.0131 \\
Hannan Quinne & 327.8416 \\
\hline
\end{tabular}

\subsubsection{Rotated Factor Loadings}

\begin{tabular}{|c|c|c|c|c|c|c|c|c|c|}
\hline \multicolumn{5}{|c|}{ Varimax Loadings } & \multicolumn{5}{|c|}{ Equamax Loadings } \\
\hline 1 & 2 & 3 & 4 & 5 & 1 & 2 & 3 & 4 & 5 \\
\hline 0.5468 & 0.0205 & 0.0155 & -0.0389 & -0.0246 & 0.5373 & 0.0112 & 0.0645 & -0.0857 & -0.0387 \\
\hline-0.1516 & 0.2139 & -0.2445 & 0.1682 & 0.0821 & -0.1097 & 0.2162 & -0.2405 & 0.1487 & 0.1575 \\
\hline 0.0075 & 0.0856 & 0.2493 & 0.0238 & -0.1009 & -0.0119 & 0.0957 & 0.2290 & 0.0377 & -0.1306 \\
\hline-0.0621 & -0.3719 & 0.0065 & 0.0877 & -0.0337 & -0.0578 & -0.3627 & -0.0060 & 0.1194 & -0.0437 \\
\hline-0.3644 & 0.0607 & -0.2408 & 0.0082 & -0.0061 & -0.3363 & 0.0649 & -0.2727 & 0.0312 & 0.0434 \\
\hline-0.0695 & -0.0030 & -0.0635 & -0.0350 & 0.3470 & -0.0711 & -0.0276 & -0.0120 & -0.0744 & 0.3450 \\
\hline-0.0196 & 0.4228 & 0.0350 & -0.0633 & -0.0276 & -0.0233 & 0.4205 & 0.0289 & -0.0820 & -0.0153 \\
\hline 0.2292 & 0.0287 & 0.0506 & 0.3203 & 0.1609 & 0.2501 & 0.0311 & 0.0991 & 0.2738 & 0.1901 \\
\hline 0.1608 & 0.1440 & -0.0744 & -0.4111 & 0.0819 & 0.1286 & 0.1134 & -0.0441 & -0.4412 & 0.0430 \\
\hline 0.0658 & 0.0316 & 0.4322 & 0.1046 & -0.0202 & 0.0340 & 0.0415 & 0.4275 & 0.1104 & -0.0750 \\
\hline \multicolumn{5}{|c|}{ Quartimax Loadings } & \multicolumn{5}{|c|}{ Orthomax Loadings } \\
\hline 1 & 2 & 3 & 4 & 5 & 1 & 2 & 3 & 4 & 5 \\
\hline 0.5476 & 0.0230 & -0.0007 & -0.0258 & -0.0269 & 0.4868 & 0.0012 & 0.2146 & 0.1232 & -0.0596 \\
\hline-0.1636 & 0.2113 & -0.2390 & 0.1755 & 0.0648 & -0.0673 & 0.2093 & -0.1250 & -0.1125 & 0.2946 \\
\hline 0.0137 & 0.0828 & 0.2530 & 0.0186 & -0.0942 & -0.0382 & 0.1173 & 0.1011 & -0.0457 & -0.2296 \\
\hline-0.0626 & -0.3746 & 0.0092 & 0.0761 & -0.0312 & -0.0370 & -0.3455 & -0.0376 & -0.1641 & -0.0445 \\
\hline-0.3720 & 0.0599 & -0.2289 & 0.0032 & -0.0129 & -0.2473 & 0.0566 & -0.3020 & -0.0428 & 0.1928 \\
\hline-0.0687 & 0.0024 & -0.0738 & -0.0218 & 0.3462 & -0.1523 & -0.0552 & 0.1455 & 0.1037 & 0.2689 \\
\hline-0.0189 & 0.4236 & 0.0374 & -0.0557 & -0.0297 & -0.0277 & 0.4122 & -0.0035 & 0.1198 & -0.0108 \\
\hline 0.2234 & 0.0241 & 0.0430 & 0.3322 & 0.1476 & 0.1722 & 0.0462 & 0.2897 & -0.2264 & 0.1320 \\
\hline 0.1684 & 0.1555 & -0.0874 & -0.3989 & 0.0922 & 0.0990 & 0.0645 & -0.0001 & 0.4618 & 0.0248 \\
\hline 0.0762 & 0.0276 & 0.4321 & 0.1005 & -0.0102 & -0.0598 & 0.0756 & 0.3040 & -0.1153 & -0.2975 \\
\hline
\end{tabular}

\subsubsection{Factor Rotation Matrix}

Varimax.

\begin{tabular}{llllll}
\hline & Factor 1 & Factor 2 & Factor 3 & Factor 4 & Factor 5 \\
\hline Factor 1 & 0.8368 & -0.0542 & 0.5388 & 0.0030 & -0.0807 \\
Factor 2 & 0.2288 & 0.7574 & -0.2605 & -0.5434 & 0.1039 \\
Factor 3 & -0.3057 & 0.6295 & 0.5173 & 0.4775 & -0.1207 \\
Factor 4 & 0.3408 & 0.1378 & -0.4437 & 0.6444 & 0.5027 \\
Factor 5 & -0.1944 & -0.0902 & 0.4210 & -0.2478 & 0.8458 \\
\hline
\end{tabular}

Equamax.

\begin{tabular}{lllccc}
\hline & Factor $\mathbf{1}$ & Factor 2 & Factor 3 & Factor 4 & Factor 5 \\
\hline Factor 1 & 0.7781 & -0.0567 & 0.5971 & -0.0437 & -0.1817 \\
Factor 2 & 0.2084 & 0.7131 & -0.2152 & -0.6236 & 0.1129 \\
Factor 3 & -0.2992 & 0.6702 & 0.4603 & 0.4903 & -0.0952 \\
Factor 4 & 0.2694 & -0.1519 & 0.5329 & -0.3171 & 0.7210 \\
Factor 5 & -0.2694 & -0.1519 & 0.5329 & -0.3171 & 0.7210 \\
\hline
\end{tabular}


Quartimax.

\begin{tabular}{llcccc}
\hline & Factor 1 & Factor 2 & Factor 3 & Factor 4 & Factor 5 \\
\hline Factor 1 & 0.8524 & -0.0541 & 0.5155 & 0.0126 & -0.0675 \\
Factor 2 & 0.2314 & 0.7730 & -0.2755 & -0.5122 & 0.1037 \\
Factor 3 & -0.3048 & 0.6136 & 0.5400 & 0.4719 & -0.1281 \\
Factor 4 & 0.3130 & 0.1323 & -0.4607 & 0.6813 & 0.4563 \\
Factor 5 & -0.1702 & -0.0752 & 0.3931 & -0.2253 & 0.8718 \\
\hline
\end{tabular}

Orthomax.

\begin{tabular}{lllccc}
\hline & Factor 1 & Factor 2 & Factor 3 & Factor 4 & Factor 5 \\
\hline Factor 1 & 0.6035 & -0.0321 & 0.6309 & 0.0717 & -0.4812 \\
Factor 2 & 0.1959 & 0.6292 & -0.0807 & 0.7191 & 0.2050 \\
Factor 3 & -0.3507 & 0.7426 & 0.2256 & -0.4544 & -0.2612 \\
Factor 4 & 0.3547 & 0.1139 & 0.3093 & -0.3855 & 0.7854 \\
Factor 5 & -0.5904 & -0.1966 & 0.6700 & 0.3501 & 0.2032 \\
\hline
\end{tabular}

3.9.3. Information Criteria

\begin{tabular}{ll}
\hline Information Criteria & Values \\
\hline Log Likelihood & -323.6940 \\
Akaike & 651.388 \\
Schwarz & 328.3067 \\
Hannan Quinne & 326.3542 \\
\hline
\end{tabular}

\section{Discussion of Results and Conclusion}

In this work, the number of variables considered was ten (10). Hence, given a sample size (n) of 30 , the values of Akaike's Information Criterion (AIC), the Schwarz Information Criterion (SIC) and the Hannan Quinne Information Criterion (HQIC) for the different number of retained factors considered are as follows; for $\mathrm{k}=2$, the AIC, SIC, and HQIC values are 241.3360,120.1451 and 119.3457 respectively. When $\mathrm{k}=3$, AIC is 236.5600 , SIC is 117.4977 and $\mathrm{HQIC}$ is 116.2985 ; and for $\mathrm{k}=5$, it shows that $\mathrm{AIC}=233.3230$, SIC $=115.3543$ and $\mathrm{HQIC}=113.3557$.

When the sample size is increased to 50, the AIC, SIC, and HQIC are 483.6900,241.5440 and 240.7657; $478.9950,239.0460$, and $237.8786 ; 484.1800,241.3374$ and 239.3919 for $\mathrm{k}=2,3$, and 5 respectively.

Finally, at the sample size of 70 , the values are AIC $=$ $660.7868, \mathrm{SIC}=330.2385$ and $\mathrm{HQIC}=329.4575$ for $\mathrm{k}=2$. When $\mathrm{k}$ is $3, \mathrm{AIC}=656.4910$, $\mathrm{SIC}=329.0131$ and $\mathrm{HQIC}=$ 327.8416 ; and finally for $\mathrm{k}=5$, the values are 651.3880 , 328.3067, and 326.3542 for AIC, SIC and HQIC respectively.

When the sample sizes are 30, and 70, the AIC, SIC, and HQIC are smallest for $\mathrm{k}=5$ followed by $\mathrm{k}=3$ and highest for $\mathrm{k}=2$. But for the sample size of 50 , the values are smallest for $\mathrm{k}=3$ followed by for $\mathrm{k}=2$ and highest for $\mathrm{k}=5$.

In conclusion, an insight into the results above shows that the optimal number of factors to retain using Principal Factors method of estimation is five (5) from the sample sizes of thirty (30) and Seventy (70); whereas for the sample size of Fifty (50), the number of factors is three(3). This conclusion is made based on the fact that in competing sets of models, the model with the smallest value of information criteria is chosen as the best model.

Also, from the vales of AIC, SIC, and HQIC obtained above, the Hannan Quinne information criterion performs best for all the three criteria considered. This is followed by the SIC and AIC respectively. Finally, we observed that the higher the sample size, the higher the value of the information criteria.

The factor rotation matrix for all the sample sizes and the number of parameters retained as considered are almost the same for all the four methods of rotation considered here.

Results above show that the values of AIC and SIC increased for all the sample sizes considered.

\section{References}

[1] Akaike, H. (1973). Information Theory and Extension of the Maximum Likelihood Principle; Second International Symposium on Information Theory (B.N. Petrov and F. Csaki, Eds.). Budapest Hungary: Akademia Kiado, 267-281

[2] Bai, J., and Serena, N.(2002). Determing the Number of Factors in Appoximate Factor Models. Econometrica $70,1,192-221$

[3] Harman, H.H. (1976). Modern Factor Analysis (3 ${ }^{\text {rd }}$ Ed.). Chicago: The University of Chicago Press.

[4] Johnson, R.A., and Wichern, D.W. (2007). Applied Multivariate Statistical Analysis ( $6^{\text {th }}$ Ed.). Prentics Hall, Englewood Cliffs, New Jersey.

[5] Lawley, D.N., and Maxwell, A.E. (1963). Factor Analysis as a Statistical Method. London: Butterworth.

[6] Onyeagu, S.I. (2003). A First Course in Multivariate Statistical Analysis ( $1^{\text {st }}$ Ed.). Mega Concept Publishers.

[7] Spearman, C. (1904). 'General Intelligence' Objectively Determined and Measured, American Journal of Psychology, 15, 201-293.

[8] Thurstone, L.L., (1947). Multiple Factor Analysis. Chicago: University of Chicago Press. 